\title{
Antitumor effect of therapeutic HPV DNA vaccines with chitosan-based nanodelivery systems
}

\author{
Alireza Tahamtan ${ }^{1,2}$, Amir Ghaemi ${ }^{1,3^{*}}$, Ali Gorji ${ }^{3,4,5,6}$, Hamid R Kalhor ${ }^{7}$, Azadeh Sajadian ${ }^{3}$, Alijan Tabarraei ${ }^{1}$, \\ Abdolvahab Moradi ${ }^{1}$, Fatemeh Atyabi $^{8}$ and Mishar Kelishadi ${ }^{1}$
}

\begin{abstract}
Background: Cervical cancer is the second-most-common cause of malignancies in women worldwide, and the oncogenic activity of the human papilloma virus types (HPV) E7 protein has a crucial role in anogenital tumors. In this study, we have designed a therapeutic vaccine based on chitosan nanodelivery systems to deliver HPV-16 E7 DNA vaccine, considered as a tumor specific antigen for immunotherapy of HPV-associated cervical cancer. We have developed a Nano-chitosan (NCS) as a carrier system for intramuscular administration using a recombinant DNA vaccine expressing HPV-16 E7 (NCS-DNA E7 vaccine). NCS were characterized in vitro for their gene transfection ability.

Results: The transfection of CS-pEGFP NPs was efficient in CHO cells and the expression of green fluorescent proteins was well observed. In addition, NCS-DNA E7 vaccine induced the strongest E7-specific CD8+ T cell and interferon $y$ responses in C57BL/6 mice. Mice vaccinated with NCS-DNA E7 vaccine were able to generate potent protective and therapeutic antitumor effects against challenge with E7-expressing tumor cell line, TC-1.

Conclusions: The strong therapeutic effect induced by the Chitosan-based nanodelivery suggest that nanoparticles may be an efficient carrier to improve the immunogenicity of DNA vaccination upon intramuscular administration and the platform could be further exploited as a potential cancer vaccine candidate in humans.
\end{abstract}

Keywords: Human papilloma virus, E7, DNA vaccine, Chitosan, Tumor

\section{Background}

Cervical cancer is the most common cancer in women worldwide and the leading cause of death from cancer among women in the developing countries of the world $[1,2]$. HPV-16 is the predominant etiologic agent of cervical cancer and encodes three transforming oncogenes, E5, E6, and E7. Their products are thus unique tumor antigens and can be used as tumor vaccines [3]. Because E6 and E7 oncoproteins are consistently retained and expressed, the E6 and E7 oncoproteins represent natural targets for anti-tumor immune response, and therefore are considered tumor-associated antigens (TAA) [4].

Several approaches have been used to develop HPV therapeutic vaccines including live vector, peptide/protein, DNA vaccine and whole cell-based approaches.

\footnotetext{
* Correspondence: ghaem_amir@yahoo.com

'Department of Microbiology, Golestan University of Medical Sciences, Gorgan, Iran

${ }^{3}$ Shefa Neuroscience Research Centre, Tehran, Iran

Full list of author information is available at the end of the article
}

These studies have shown the importance of T-cell responses in protecting against tumors in human and animal models [5]. Of these approaches, DNA vaccine targeting the E7 antigen offers a potentially effective approach to control E7-expressing tumors $[4,6,7]$.

DNA vaccines represent a valid approach to the generation of antigen-specific immunotherapy for several reasons $[8,9]$. The plasmid vectors are safe, have low immunogenicity, and can be repeatedly administered. Additionally, DNA vaccines can be easily prepared in large scale with high purity and are highly stable compared to viral vectors [10]. DNA vaccines have also demonstrated to generate both humoral and cell mediated immune responses [11,12].

However, there are several drawbacks preventing the clinical application of DNA vaccines [13]. After intramuscular administration, it is difficult for the vaccines to move through cell membranes, so only a small amount reaches antigen presenting cells (APC) to induce immune responses $[14,15]$. 
Although the reasons for lack of potency of DNA vaccines in human subjects and non-human primates remain poorly understood [10], there is a clear need to improve the transfection efficiency in vivo for allowing much lower dose of plasmid DNA.

Novel delivery systems for administration may be keys to address this need. Among possible delivery systems, chitosan nanoparticles hold promise because of their ability to protect encapsulated nucleic acid-based antigens, and to promote delivery of adsorbed DNA to antigen-presenting cells $[13,16]$.

Chitosan (CS) is a non-toxic biodegradable and biocompatible polycationic polymer, which can bind and protect the entrapped DNA from degradation by nuclease and increase the efficiency of cellular DNA uptake [16-18], additionally CS exhibit potential adjuvant properties, such as promoting endocytotic uptake and elevating immune responses [19].

Zaharoff et al. have demonstrated that chitosan solution improved antigen-specific antibody and antigenspecific splenic $\mathrm{CD} 4+$ proliferation responses to a subcutaneous vaccination with a model protein antigen in the absence of additional adjuvants [20]. These studies established a basis for the use of chitosan nanoparticles as a DNA vaccine delivery system.

In this study, we have evaluated the immune response elicited in C57BL/6 mice by Nano-chitosan (NCS) containing a recombinant DNA vaccine expressing HPV-16 E7, as well as the levels of protection against TC-1 tumor cell challenge, following intramuscular administration.

\section{Methods}

\section{Mice and cells}

Female 6-8-week-old C57BL/6 mice were obtained from the Institute Pasteur of Iran (Karaj, Iran). Mice were housed for 1 week before the experiment, given free access to food and water and maintained in a light/dark cycle. All experiments were carried out in accordance with the Animal care and use protocol of Golestan University of Medical Sciences of Iran.

The production and maintenance of TC-1 cells have been described previously [12]. The cells were C57BL/6 mouse lung endothelial cells transformed with HPV 16 E6 and E7 oncogenes and an activated H-ras gene.

The in vitro transient transfection studies were performed in Chinese hamster ovary ( $\mathrm{CHO})$ (Pasteur Institute of Iran) cell line. The $\mathrm{TC}-1$ and $\mathrm{CHO}$ cell lines were cultured in RPMI 1640 medium (Gibco-BRL, UK) supplemented with 10\% FBS (Gibco), $1 \mathrm{mM}$ sodium pyruvate (Sigma), $100 \mathrm{U} / \mathrm{ml}$

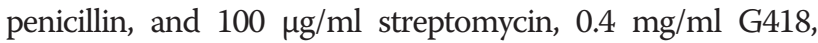
and $0.2 \mathrm{mg} / \mathrm{ml}$ hygromycin at $\mathrm{pH} 7.2$ at $37^{\circ} \mathrm{C}$ with $5 \% \mathrm{CO}_{2}$.

\section{Plasmid construction}

The generation of pcDNA3.1-E7 has been described previously. Plasmid constructs were confirmed by DNA sequencing and expression. Amplification and purification of DNA were previously described [6]. Stocks of endotoxin free DNA vaccine plasmids and vector control plasmid (pcDNA3.1) in 0.1 M PBS were prepared for invivo immunization studies using the EndoFree ${ }^{\oplus}$ Plasmid Maxi Kit (Qiagen, Hilden, Germany) and dissolved in endotoxin-free Tris-EDTA (Sigma, St. Louis, MO).

\section{Preparation and characterization of nano-chitosan-based DNA vaccine}

Chitosan (CS) with the deacetylation degree of $95 \%$ and the molecular weight (MW) of $360 \mathrm{kDa}$ was purchased from the Primex (Karmoy, Norway) and depolymerized by a chemical reaction as previously described [21] to obtain the low molecular weights of CS. The molecular weight of depolymerized CS was determined by gel permeation chromatography (GPC) using pullulane standards [22]. The Nano-chitosan (NCS) were prepared according to the ionotropic gelation method based on the interaction between the negative groups of the pentasodium tripolyphosphate (TPP) cross-linker (Merck, Darmstadt Germany) and the positively charged amino groups of NCS as described previously [23]. The complexes were allowed to stand at room temperature for $30 \mathrm{~min}$ and stored at $4^{\circ} \mathrm{C}$ until use.

The hydrodynamic mean diameter of prepared chitosan nanoparticles was determined by dynamic light scattering using Nano-Zetasizer (Malvern Instruments, Worcestershire, United Kingdom) with a wavelength of $633 \mathrm{~nm}$ at $25^{\circ} \mathrm{C}$ with an angle detection of 90 degrees. The samples were diluted with freshly filtered deionized water and all measurement was done three times. The zeta potential of prepared chitosan nanoparticles was determined by laser Doppler electrophoresis using Zetasizer (Nano-ZS Malvern Instruments). All samples were diluted (1:5) in deionized water and each sample was measured three times.

NCS -DNA vaccine complex were prepared as previously described [24]. Briefly, the complex formation between NCS and pDNA at different N/P ratios were analyzed by $0.8 \%$ agarose gel electrophoresis in TBE buffer along with naked pDNA as control. Sample was analyzed using electrophoresis at $40 \mathrm{~V}$ for $60 \mathrm{~min}$. The in vitro transfection study was performed using $\mathrm{CHO}$ cell line and pEGFP-N1 (Clontech Laboratories) as marker gene. EGFP expression in cells investigated through fluorescence microscopy. To evaluate the expression of NCSDNA vaccine encoding HPV-16 E7 gene (NCS-DNA E7) in the CHO cells, SDS-PAGE and Western blot analysis using monoclonal mouse anti-HPV E7 antibody were used. Briefly, the extracted total proteins lysed in SDSPAGE sample loading buffer, and lysates separated by SDS-PAGE. Separated proteins by SDS-PAGE, transferred onto PVDF, and E7 protein was detected using anti-E7 monoclonal antibody (Abcam, UK). The specific band was 
detected with goat anti-mouse secondary antibody (Sigma, St Louis, MO) and staining with diaminobenzidine (DAB) substrate. The TC-1 cell line and cells transfected with Lipofectamin were used as controls.

\section{Tumor therapy assay}

For in vivo therapeutic experiments, C57BL/6 mice were divided into six groups $(n=10)$. The mice were challenged by subcutaneous (S.C) injection in the right flank with $2 \times 10^{5}$ TC- 1 cells suspended in $100 \mu \mathrm{l} \mathrm{PBS}$. After one week, the mice were immunized intramuscularly with $90 \mu \mathrm{g}$ naked DNA vaccine encoding HPV-16 E7 (DNA-E7) thrice at 7-day intervals (group 1).

Each animal in Groups II and III were respectively administered with $90 \mu \mathrm{g}$ NCS-DNA E7 and NCS-pcDNA3.1 plasmid with the same protocol.

Blank Nano-chitosan (NCS), PBS and pcDNA3.1 were injected according to the same protocol into the IV, V and VI group of mice as control groups (NCS, PBS and $\mathrm{pcDNA}_{3}$ groups).

Subcutaneous tumor volume was estimated according to Carlsson's formula [12]. Hence, the largest (a) and the smallest (b) superficial diameters of the tumor were measured in a blinded, coded fashion twice a week and then the volume $(\mathrm{V})$ of the tumor was calculated $(\mathrm{V}=\mathrm{a} \times$ $\mathrm{b} \times \mathrm{b} / 2$ ). Statistical analysis was performed using Student's $t$ test. All values were expressed as means \pm S.D.

Five mice per group were sacrificed one week following the third immunization and the spleens were removed aseptically, and then cell proliferation, cytolytic activity and cytokine secretion were assayed. All tests were performed in triplicate for each mouse. Results are representative of three independent experiments.

\section{Lymphocyte proliferation assay (LPA)}

One week after the third immunization, 5 mice per group were sacrificed and their splenocytes were obtained and treated with ammonium chloride-potassium lysis buffer for $1 \mathrm{~min}$ to deplete erythrocytes. In 96-well flat-bottom culture plates (Nunc, Denmark) splenocytes $\left(2 \times 10^{5}\right.$ per well) were cultured in triplicate with RPMI1640 supplemented with $10 \%$ fetal calf serum, 1\% L-glutamine, $1 \%$ HEPES, $0.1 \%$ penicillin/streptomycin. The cultured cells were incubated with $4 \times 10^{5} \mathrm{TC}-1$ cells previously treated with mitomycin C (Sigma, St. Louis, Mo) $(30 \mu \mathrm{g} / \mathrm{ml}$ for $3 \mathrm{~h}$ ), T cell mitogen PHA (phytohemagglutinin, positive control), $2 \mu \mathrm{g} / \mathrm{ml}$ of BSA (irrelevant antigen), or medium (negative control) per well at $37^{\circ} \mathrm{C}$ in $5 \% \mathrm{CO}_{2}$.

After 3 days, MTT (3-(4,5-dimethyl tetrazolyl-2) 2,5 diphenyl) tetrazolyumbromide (Sigma chemicals) in concentration of $5 \mu \mathrm{g} / \mathrm{ml}$ was added per well and incubated for $5 \mathrm{~h}$ at $37^{\circ} \mathrm{C}$ in $5 \% \mathrm{CO}_{2}$. DMSO (dimethyl sulfoxide)
$(100 \mu \mathrm{l})$ was added to dissolve produced formazan crystals.

Plates were read at $540 \mathrm{~nm}$, and the results were expressed as stimulation index (SI). The SI was determined as follows: OD values of stimulated cells minus relative cell numbers of unstimulated cells divided by relative OD values of unstimulated cells.

All tests were performed in triplicate for each mouse.

\section{CD8 cytotoxicity assay}

One week after the third immunization, for each sample obtained from individual mouse (five mice per group), single cell suspension of mononuclear cells (used as the effecter cells) were cocultured in phenol red-free RPMI containing 3\% FCS with washed target cells EL4 at different effector-to-target cell $(\mathrm{E} / \mathrm{T})$ ratio $(25: 1,50: 1$ and 100:1). For preparation of the target cells, EL-4 cells were stimulated with $4 \times 10^{5} \mathrm{TC}-1$ cells previously treated with mitomycin $\mathrm{C}(30 \mu \mathrm{g} / \mathrm{ml}$ for $3 \mathrm{~h})$ and then incubated for $4 \mathrm{~h}$.

After centrifugation, the supernatants $(50 \mu \mathrm{l} / \mathrm{well})$ were transferred to the 96-well flat-bottom plates, and lyses of target cells were determined by measuring LDH release using Cytotoxicity Detection Kit (LDH) according to the procedures stated by the manufacturer (Takara Company).

Several controls were used. The 'high control' was used for measuring total LDH release from the target cells (all EL4 cells were lysed with medium containing 1\% Triton X-100). The 'low control' was used for measuring natural release of LDH from the target cells (which was obtained by adding EL4 cells only to the assay medium).

The optical density was read at $490 \mathrm{~nm}$ after $30 \mathrm{~min}$ incubation at room temperature and cytotoxicity was calculated according to the formula:

$$
\begin{aligned}
\% \text { Cytotoxicity }= & (\text { Experimental value }- \text { Low control } / \\
& \text { High control }- \text { Low control }) \times 100
\end{aligned}
$$

\section{Cytokine assay}

One week after third immunization, splenocytes were obtained from five immunized mouse of each group and used for the IFN- $\gamma$ and IL-4 production assay. Briefly, $5 \times 10^{5}$ cells/well in the presence of $4 \times 10^{5} \mathrm{TC}-1$ cells previously treated with mitomycin $\mathrm{C}(30 \mu \mathrm{g} / \mathrm{ml}$ for $3 \mathrm{~h}$ ) were harvested in 96-well flat-bottom plates with complete RPMI 1640 supplement with 10\% FCS, 1\% L-glutamine, 1\% HEPES, $0.1 \%$ 2-mercaptoethanol and $0.1 \%$ penicillin/ streptomycin. Cells were cultured for 2 days at $37^{\circ} \mathrm{C}$ in $5 \%$ $\mathrm{CO}_{2}$. Supernatants were then collected and analyzed for the presence of IFN- $\gamma$ and IL-4 using ELISA commercial kits according to the manufacturers protocol (eBioscience, 
Inc. San Diego, CA). All tests were performed in triplicate for each mouse (Five mice per group).

\section{Statistical analysis}

Lymphocyte proliferation, CTL and cytokine assay were analyzed by a one-way ANOVA. Significant differences of tumor growth on given days were assessed by Student's $t$-test. Differences were considered statistically significant when $\mathrm{P}$ value $<0.05$. All tests were performed in triplicate and all data are expressed as mean \pm SD.

\section{Results}

\section{Characterization of chitosan-based nanodelivery systems for E7 DNA vaccine}

The particle size of the NCS ranged between 40 and $150 \mathrm{~nm}$; nanoparticles showed a narrow size distribution (PDI: 0.149), positive zeta potential with a mean diameter of about $70 \mathrm{~nm}$.

The mean zeta potential of prepared NPs before packaging was approximately $+20 \mathrm{mV}$.

NCS/pDNA complexes were developed by adding the pDNA solution to the prepared nanoparticle's solution. The complex formation was confirmed by gel electrophoresis [24].

The in vitro transfection study was performed using $\mathrm{CHO}$ cell line and EGFP-N1 as marker gene through fluorescence microscopy. EGFP expression was observed in $\mathrm{CHO}$ cells transfected with NCS - pEGFP in a similar extent to the control cells which were transfected with lipofectamine (Figure 1A).
To evaluate the expression of HPV-16 E7 gene in the $\mathrm{CHO}$ cells, Western blot analysis using monoclonal mouse anti-HPV E7 antibody was used. The TC-1 cell line and cells transfected with Lipofectamine were used as positive controls. The analysis showed an expression of HPV-16 E7 11 KDa from NCS-DNA E7 in the Western blot according to the TC-1 cells and cells transfected with Lipofectamine (Figure 1B).

\section{Lymphocyte proliferation response to E7 antigen}

One week after the third immunization, the splenocytes from the immunized mice were harvested and restimulated in vitro with E7 antigen for the lymphocyte proliferation assay.

As shown in Figure 2, the lymphocyte proliferation was significantly higher in mice treated with the NCSDNA E7 group than in those treated with DNA-E7 and control groups (DNA-E7, NCS, NCS-pcDNA3.1, PBS and pcDNA3.1) $(\mathrm{P}<0.001)$. As it appeared, there is no (statistically) significant difference among control groups.

\section{Induction of antigen specific CD8+ CTL activity}

In order to analyze the capacity of HPV-16 E7 to enhance the E7-specific CD8 + cytotoxic T-lymphocyte (CTL) response, the response in immunized mice was examined using the LDH release assay. The LDH release increased with the E:T cell ratio up to the maximum ratio of 100:1 used in the present study. As shown in Figure 3, the cytolytic activity was significantly higher in mice treated with the NCS-DNA E7, with almost $50 \%$ specific lysis at 100:1 $\mathrm{E} / \mathrm{T}$ ratio, than in those in

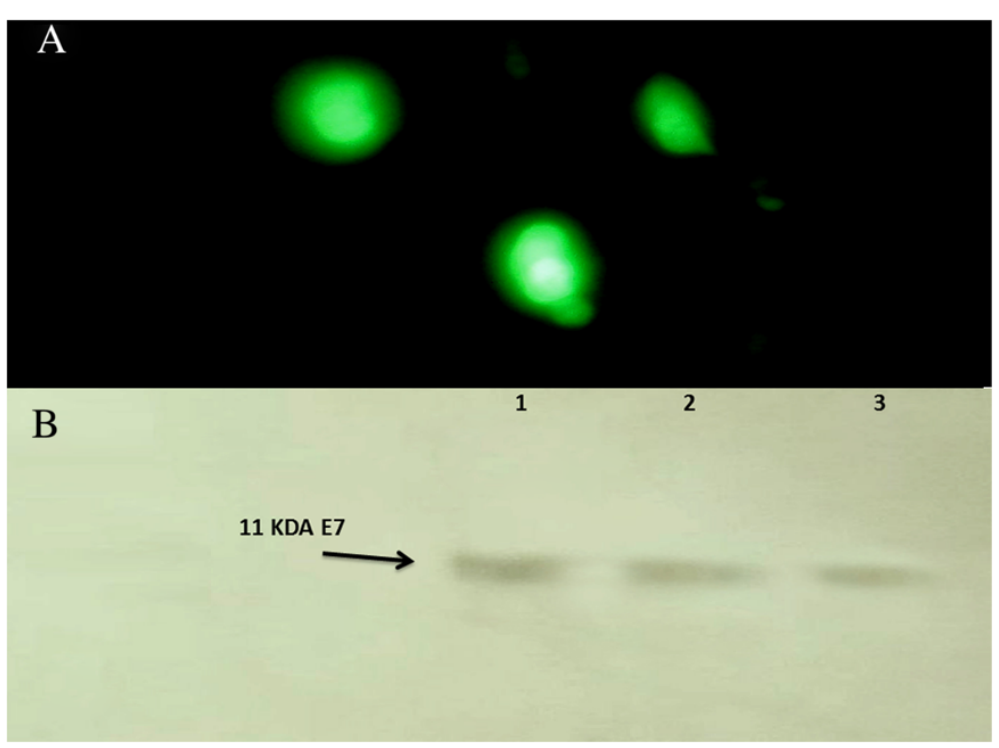

Figure 1 Transfection analysis of the chitosan nanodelivery systems. A) CHO cells transfected by NCS - pEGFP; B) Western blot analysis of HPV E7 antigen expression by NCS-DNA E7. Cell extracts or supernatants were prepared as described in Methods; proteins were separated by SDS polyacrylamide gel electrophoresis, blotted on PVDF membranes, and incubated with the specific anti-HPV-16 E7 monoclonal antibody. The arrow indicates the E7 protein of approximately $11 \mathrm{KDa}$ of molecule weight. Lane 1: Lysate from TC-1 cells, Lane 2: pcDNA3.1/E7 with Lipofectamine, lane 3: NCS-DNA E7. 


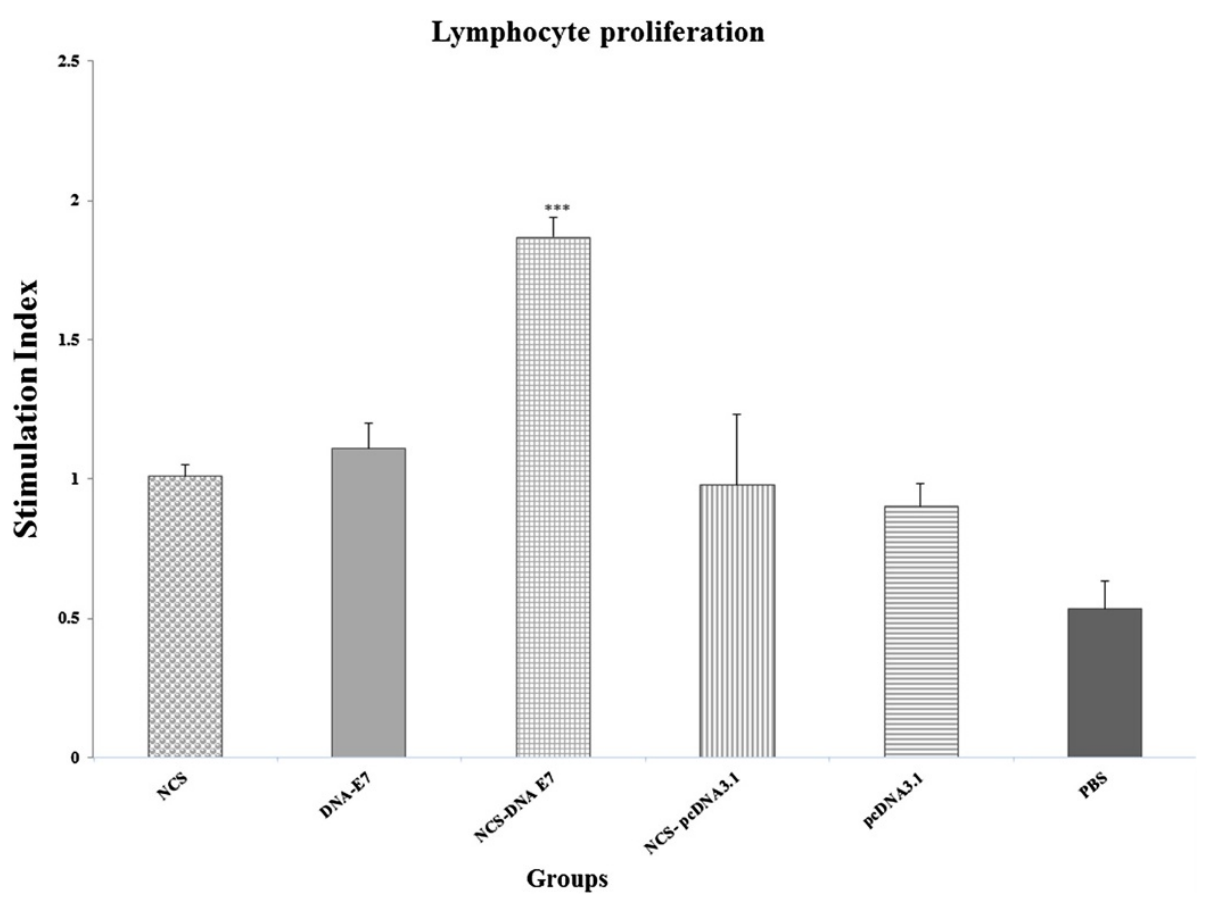

Figure 2 Splenocyte proliferation levels after in vitro stimulation with HPV-16 E7 antigen. The mice were injected intramuscularly thrice at 7-day intervals with different Nano-chitosan (NCS) regimes. One week after final immunization, spleens of individual mice (five per group) were removed and lymphocyte proliferation was evaluated with MTT method. Formazan crystals were dissolved in dimethyl sulfoxide and optical densities were read at $540 \mathrm{~nm}$. Values are the mean \pm standard error of the mean for the experiments. *** Indicates statistically significant difference between the NCS-DNA E7 group as determined by one-way ANOVA $(P<0.001)$ with other groups.

other groups (DNA-E7, NCS, NCS-pcDNA3.1, PBS and pcDNA3.1) $(\mathrm{P}<0.001)$. Furthermore, DNA-E7 $(\sim 30 \%$ at 100:1 E/T ratio), NCS-pcDNA3.1 ( 20\% at 100:1 E/T ratio) and NCS ( 17\% at 100:1 E/T ratio) groups had a significantly higher antigen-specific CTL response than the group treated with PBS $(\mathrm{P}<0.05)$.

\section{Cytokine assay}

We evaluated the E7-specific IFN- $\gamma$ (representative of a Th1 immune response) and IL-4 (representative of a Th2 immune response) in splenocyte culture supernatants from mice vaccinated three times with DNA vaccine encoding HPV-16 E7 with or without chitosan nanodelivery.

As shown in Figure 4A, B, mice immunized with the NCS-DNA E7 produced significantly more IFN- $\gamma$ and IL-4 than other immunized mice $(\mathrm{P}<0.001)$ and lymphocytes from the NCS-DNA E7 group produced the largest amounts of IFN- $\gamma$ and IL-4. Furthermore, the DNA-E7 vaccine stimulated IFN- $\gamma$ production more than pcDNA3.1 and PBS groups $(\mathrm{P}<0.05)$.

\section{Reduction of tumor volume by therapeutic immunization} To determine whether the observed increase in E7-specific immunity could be converted into a better E7-specific therapeutic effect, we designed an in vivo tumor therapy experiment via an E7-expressing murine tumor cell line, TC-1, as a model of cervical carcinoma.

We investigated whether administration of NCS-DNA E7 vaccine could lead to regression of preexisting tumors. For this porpuse, TC- 1 cells were first injected into $\mathrm{C} 57 \mathrm{BL} / 6$ mice at a dose of $2 \times 10^{5} \mathrm{TC}-1$ cells in the right flank. One week later, each mouse was immunized with either NCS-DNA E7, E7 DNA, NCS, NCS-pcDNA3.1, PBS and pcDNA3.1 thrice at 7-day intervals. The tumors were measured twice a week once they became palpable. The tumor volume was monitored up to 6 weeks after the tumor challenge.

As shown in Figure 5, six weeks after tumor induction, the final tumor volume were significantly reduced $52 \%$ by NCS-DNA E7 (from 1.2 to $0.8 \mathrm{~mm}^{3}$ ) and $60 \%$ by E7 DNA (from 1.2 to $0.95 \mathrm{~mm}^{3}$ ) compared to pcDNA3 or PBS control values $(\mathrm{P}<0.001)$. Additionally, the average tumor volume in the NCS-DNA E7 group was significantly lower than that in the E7 DNA group $(\mathrm{P}<0.01)$.

As control for nanodelivery, we also have evaluated antitumor effects of NCS and NCS-pcDNA3.1. The statistical analysis of tumor volumes did not reveal significant difference between these groups and PBS and pcDNA3.1 groups $(\mathrm{P}>0.05)$.

In summary, these results showed that chitosan-based nanodelivery systems for E7 DNA vaccine could significantly 


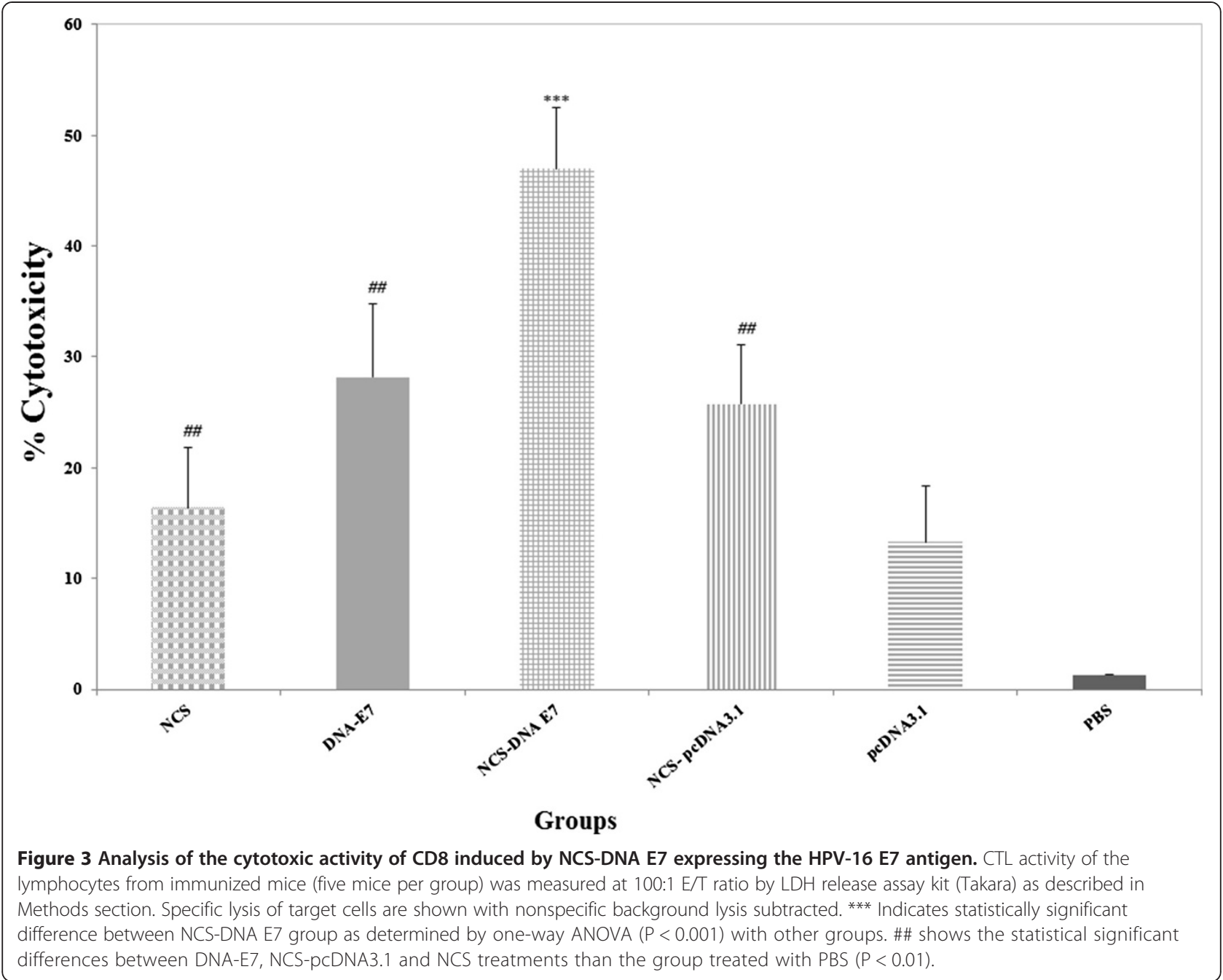

reduce tumor volume and eradicate the established E7expressing tumors. This indicated that chitosan-based nanodelivery significantly promoted antitumor immunity of DNA vaccine.

\section{Discussion}

Cancer immunotherapy using DNA vaccines have emerged as a potentially promising approach for the control of tumors. The main purpose of this study was to develop an HPV-16 DNA vaccine that could express the E7 antigen and enhance cellular immune response compared to the naked DNA vaccine. With regard to the application of DNA vaccines, if the delivery system can be directed to APC and induce DNA vaccine release in endolysosomes, an effective T-cell immunity may be achieved at lower doses of plasmid DNA, which is safer and more costeffective [25].

Several studies have investigated the use of chitosan as a gene delivery system, as it has shown rapid degradation in lysosomal compartment after cellular uptake, allowing for enhanced MHC-I restricted antigen presentation [4]. In mice, DNA vaccines have been delivered orally using a variety of carriers. One recent study has shown that oral gene delivery with chitosan-DNA nanoparticles was able to generate a higher level expression of gene in vivo [26].

Khatri et al. evaluated intranasal administration of chitosan pDNA nanoparticles expressing the surface antigen of hepatitis B virus (HBsAg) for immunization against hepatitis B in Balb/c mice model demonstrating successful generation of a systemic humoral and cellular immune response [27].

In addition to the oral and intranasal vaccination routes, chitosan-based systematic vaccine administration routes such as subcutaneous and intramuscular have also been explored for immunization. However, there are very limited examples where chitosan has been applied as DNA vaccine carriers for systemic vaccination.

Zaharoff et al. showed that chitosan dissolved in buffer pH 6.2 could be used as an adjuvant to enhance both 

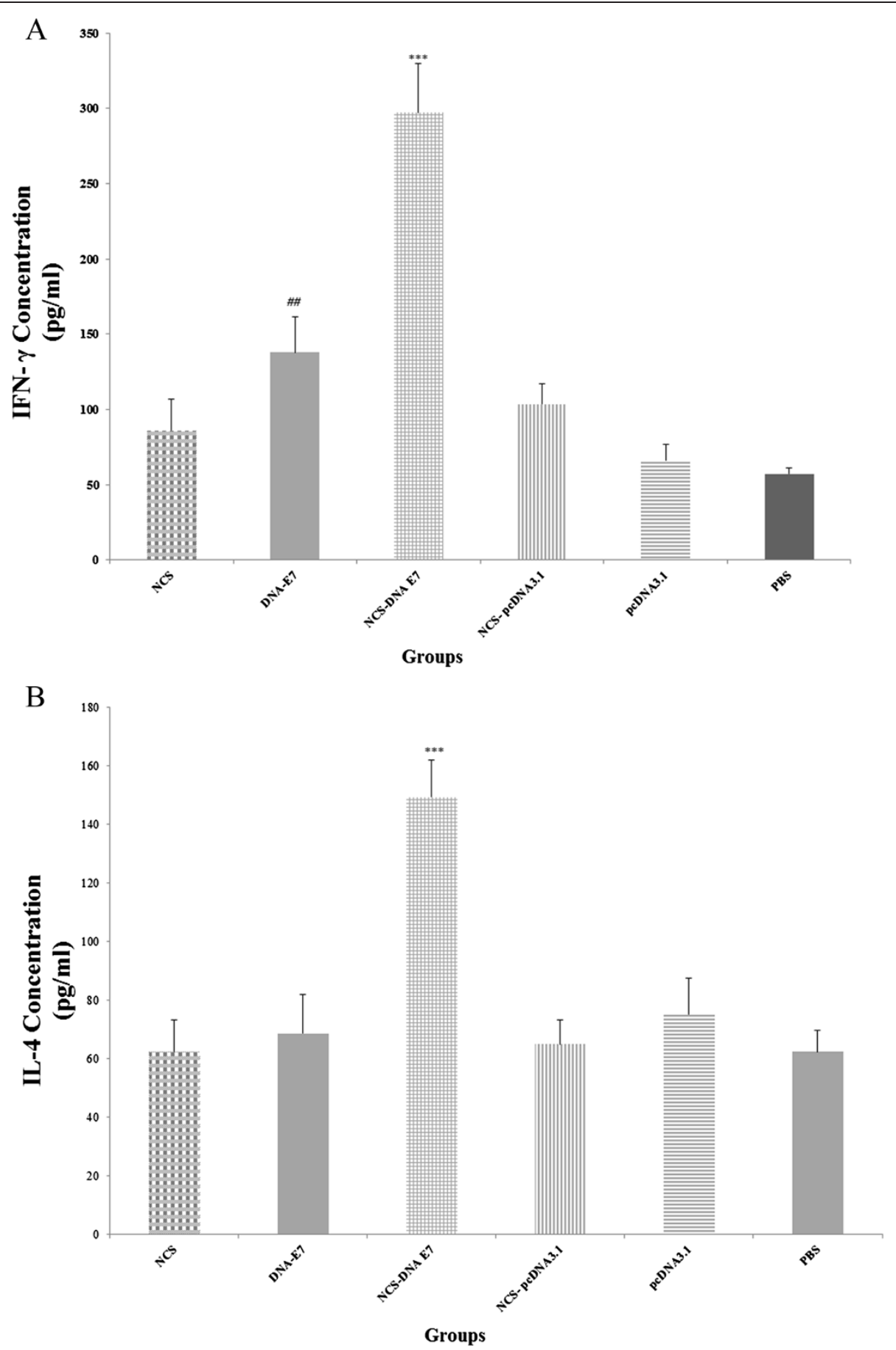

Figure 4 Concentration of IFN- $\gamma$ and IL-4 in supernatant following stimulation of cultured splenocytes with $4 \times 10^{5}$ TC- 1 cells previously treated with mitomycin C. Data presented as means \pm standard error for five mice per group. ${ }^{* * *}$ Indicates statistically significant difference $(P<0.001)$ between NCS-DNA E7 group as determined by one-way ANOVA $(P<0.01)$ with other groups $(\mathbf{A}, \mathbf{B})$. \#\# indicates the statistical significant differences between DNA-E7 treatment and pcDNA3.1 and PBS groups $(P<0.01)(\mathbf{A})$.

cell-mediated and humoral immune responses [20]. Illum et al. evaluated a flu vaccine, formulated as a chitosanDNA nanoparticle complex. Intramuscular or intranasal administration of the complex in BALB/c mice resulted in antibody titers that were elevated when compared to those of naked DNA [16].

In present study, we have produced NCS of fewer than $100 \mathrm{~nm}$ diameter to facilitate the entrance of the particles 


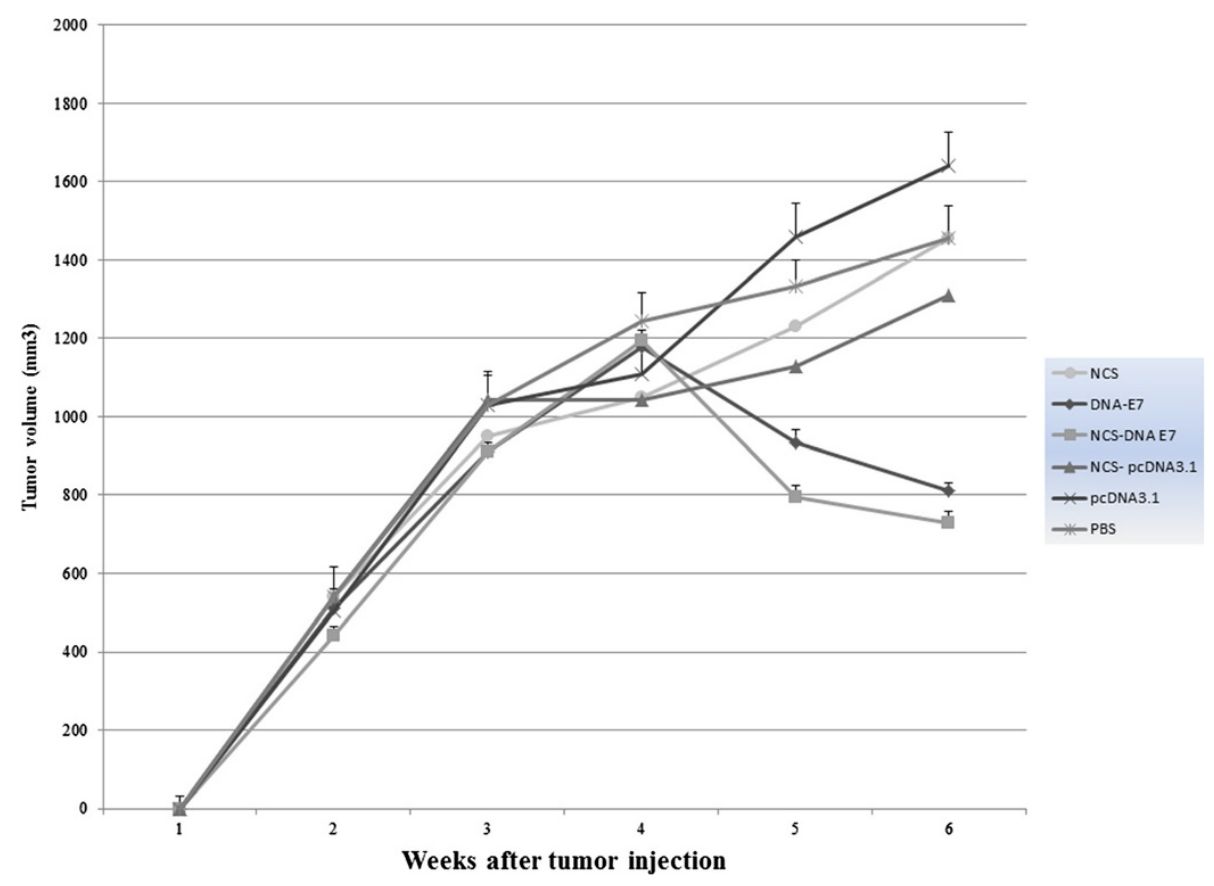

Figure 5 In vivo antitumor effects generated by treatment with NCS-DNA E7, DNA-E7, NCS-pcDNA3.1 and NCS. C57BL/6 mice were inoculated with $2 \times 10^{5} \mathrm{TC}-1$ tumor cells subcutaneously. Mice were then treated with Nano-chitosan (NCS) and DNA regimes as described in Methods. Mice were monitored for tumor growth by measuring diameters with calipers twice a week. Line and scatter plot graphs depicting the tumor volume (in $\mathrm{mm}^{3}$ ) are presented. The data presented are a representation of three independent experiments.

to the cells. The resultant NCS showed mean diameter of about $70 \mathrm{~nm}$ with spherical shape and a narrow size distribution. To evaluate the therapeutic efficacy of chitosan-based nanodelivery systems in vivo, we investigate the efficiency of intramuscular administration of encapsulated DNA vaccine in NCS, as carrier molecules for HPV-16 DNA vaccine construct, carrying the most immunogenic oncogenes of HPV-16 in mouse model for cervical cancer (TC-1 cells). The results demonstrated the induction of effective cellular immunity and antitumor response in immunized mice and supported the feasibility of Nano-chitosan to be explored for enhancing the immune responses of the DNA vaccine.

In agreement with the results of the present study, it has been previously shown that after intramuscular administration of pVAX hepatitis B virus core ( $\mathrm{HBc})$ DNAnanoparticle complex as vaccine delivery systems in C57BL/6 mice, a much stronger immune response was elicited than for the vaccine alone, shown by elevated antibody production, higher level of IFN- $\gamma$ secretion, and augmented Hbc Ag-specific cytotoxic T-lymphocyte response in murine splenocytes [28].

Additionally, Zhao and colleagues have showed that chitosan nanoparticles containing the DNA vaccine induced significantly swine influenza -specific cell-mediated and humoral immunity in mice [29].
Moreover, present findings are in parallel with previous studies from Zhou et al. that investigated the potency of the Chitosan as vectors for the delivery of plasmid DNA. After delivery by intramuscular immunization in $\mathrm{BALB} / \mathrm{c}$ mice, the Chitosan induced an enhanced serum antibody response 10 times more potent than naked DNA vaccine. Additionally, in contrast to naked DNA, the Chitosan induced potent cytotoxic $\mathrm{T}$ lymphocyte responses at a low dose [30].

In other studies for evaluation of HPV-16 E7 as model antigen in the development of a therapeutic DNA vaccine candidate, several DNA vaccines against human papillomavirus (HPV) related malignancies have been performed. Our previous findings demonstrated that DNA vaccines encoding HPV-16 E7 could enhance the induction of antigen-specific cytotoxic CD8+ T cell responses and IFN- $\gamma$ and confer protective immunity and therapeutic control of tumor growth $[6,12]$. In a trial for anal dysplasia, encapsulated plasmid DNA encoding HLA-A2 epitopes from HPV16 E7 protein in polymer microparticles administrated intramuscularly and induced enhanced $\mathrm{T}$ cell responses in 10 of 12 patients [31]. Therefore, induction of both CD4+ T cells secreting Th1-type cytokines and CD8+ cytotoxic T cells play important effector roles for vaccines aiming therapeutic activation of protective antitumor immunity, similar to what was shown by the present NCS-DNA E7 vaccine. 
Thus, it was reasonably believed that Chitosan played a key role in supporting cellular uptake by cell-Chitosan interactions and enhanced the antigen presentation.

\section{Conclusions}

Taken together, our study provides important insights for eradicating HPV-induced cancers exploiting a ChitosanBased Nanodelivery. Through this new approach, the bioavailability and stability of the vaccine were significantly improved, leading to induction of antigen-specific CD8+ cytotoxic T lymphocytes (CTL), splenic CD4+. Ultimately, the delivered vaccine induced production of IFN- $\gamma$ to promote antitumor immunocytotoxic response and reduce the tumor volumes.

\section{Abbreviations}

HPV: Human papilloma virus; NCS: Nano-chitosan; CHO: Chinese hamster ovary; TPP: Pentasodium tripolyphosphate; TPP: Pentasodium tripolyphosphate; PHA: Phytohemaglotinin; APC: Antigen-presenting cell; CTL: Cytolytic T Iymphocyte; IFN- $\gamma$ : Interferon $\gamma$; LL-4: Interleukin 4; LDH: Lactate dehydrogenase; MTT: 3-(4,5-Dimethylthiazol-2-YI)-2,5-diphenyltetrazolium bromide; thiazolyl-blue; OD: Optical density; FBS: Fetal bovine serum; DMSO: Dimethyl sulfoxide; RPMI: 1640 Roswell Park Memorial Institute (name of the medium); Th: T helper.
}

\section{Competing interests}

All authors declare that they have no competing interests.

\section{Authors' contributions}

AT, did most of the experiments, AG, FA and HK participated in the design of the study, AG and AT drafted the manuscript, and AG, AT, AS and AM conceived of the study and data interpretation, MK and AS gave some suggestions. All authors read and approved the final version of the manuscript.

\section{Acknowledgements}

The authors appreciate the financial support of the Research Deputy at Golestan Medical University. This project was extracted from a MSc thesis.

\section{Author details}

'Department of Microbiology, Golestan University of Medical Sciences, Gorgan, Iran. ${ }^{2}$ Department of Virology, School of Public Health, Tehran University of Medical Sciences, Tehran, Iran. ${ }^{3}$ Shefa Neuroscience Research Centre, Tehran, Iran. ${ }^{4}$ Institut für Physiologie I, Westfälische Wilhelms-Universität Münster, Robert-Koch-Strasse, 48149 Münster, Germany. ${ }^{5}$ Klinik und Poliklinik für Neurochirurgie, Westfälische Wilhelms-Universität Münster, Münster 48149, Germany. ${ }^{6}$ Department of neurology, Westfälische Wilhelms-Universität Münster, Münster, Germany. ${ }^{7}$ Biochemistry Research laboratory, Department of Chemistry, Sharif University of Technology, Tehran, Iran. ${ }^{8}$ Nanotechnology Research Centre, Faculty of Pharmacy, Tehran University of Medical Sciences, Tehran, Iran.

Received: 9 May 2014 Accepted: 22 July 2014

Published: 31 July 2014

\section{References}

1. Ma B, Maraj B, Tran NP, Knoff J, Chen A, Alvarez RD, Hung CF, Wu TC: Emerging human papillomavirus vaccines. Expert Opin Emerg Drugs 2012, 17(4):469-492.

2. Stanley MA: Genital human papillomavirus infections: current and prospective therapies. J Gen Virol 2012, 93(Pt 4):681-691.

3. Lin K, Roosinovich E, Ma B, Hung CF, Wu TC: Therapeutic HPV DNA vaccines. Immunol Res 2010, 47(1-3):86-112.

4. Pang $\mathrm{CL}$, Thierry F: Human papillomavirus proteins as prospective therapeutic targets. Microb Pathog 2013, 58:55-65.
5. Su JH, Wu A, Scotney E, Ma B, Monie A, Hung CF, Wu TC: Immunotherapy for cervical cancer: research status and clinical potential. BioDrugs 2010, 24(2):109-129.

6. Ghaemi A, Soleimanjahi H, Gill P, Hassan Z, Jahromi SR, Roohvand F: Recombinant lambda-phage nanobioparticles for tumor therapy in mice models. Genet Vaccines Ther 2010, 8:3.

7. Morrow MP, Yan J, Sardesai NY: Human papillomavirus therapeutic vaccines: targeting viral antigens as immunotherapy for precancerous disease and cancer. Expert Rev Vaccines 2013, 12(3):271-283.

8. Naderi M, Saeedi A, Moradi A, Kleshadi M, Zolfaghari MR, Gorji A, Ghaemi A: Interleukin-12 as a genetic adjuvant enhances hepatitis C virus NS3 DNA vaccine immunogenicity. Virol Sin 2013, 28(3):167-173.

9. Sajadian A, Tabarraei A, Soleimanjahi H, Fotouhi F, Gorji A, Ghaemi A: Comparing the effect of Toll-like receptor agonist adjuvants on the efficiency of a DNA vaccine. Arch Virol 2014. doi:10.1007/s00705-014-2024-4.

10. Li L, Saade F, Petrovsky N: The future of human DNA vaccines. J Biotechnol 2012, 162(2-3):171-182.

11. Ghaemi A, Soleimanjahi H, Bamdad T, Soudi S, Arefeian E, Hashemi SM, Ebtekar M: Induction of humoral and cellular immunity against latent HSV-1 infections by DNA immunization in BALB/c mice. Comp Immunol Microbiol Infect Dis 2007, 30(4):197-210.

12. Ghaemi A, Soleimanjahi H, Gill P, Hassan ZM, Razeghi S, Fazeli M, Razavinikoo SM: Protection of mice by a lambda-based therapeutic vaccine against cancer associated with human papillomavirus type 16. Intervirology 2011, 54(3):105-112.

13. Alpar HO, Papanicolaou I, Bramwell WW: Strategies for DNA vaccine delivery. Expert Opin Drug Deliv 2005, 2(5):829-842.

14. Johnston SA, Qu BX, McGuire M, Stemke-Hale K, Sykes K: Applications of, and future challenges for, genetic vaccines. Dev Biol (Basel) 2000, 104:3-8.

15. Pachuk $C$, , McCallus DE, Weiner DB, Satishchandran C: DNA vaccines-challenges in delivery. Curr Opin Mol Ther 2000, 2(2):188-198.

16. Illum L, Jabbal-Gill I, Hinchcliffe M, Fisher AN, Davis SS: Chitosan as a novel nasal delivery system for vaccines. Adv Drug Deliv Rev 2001, 51(1-3):81-96.

17. Bolhassani A, Javanzad S, Saleh T, Hashemi M, Aghasadeghi MR, Sadat SM: Polymeric nanoparticles: potent vectors for vaccine delivery targeting cancer and infectious diseases. Hum Vaccin Immunother 2013, 10(2):2013.

18. Masotti A, Ortaggi G: Chitosan micro- and nanospheres: fabrication and applications for drug and DNA delivery. Mini Rev Med Chem 2009, 9(4):463-469.

19. Feng G, Jiang Q, Xia M, Lu Y, Qiu W, Zhao D, Lu L, Peng G, Wang Y: Enhanced immune response and protective effects of nano-chitosan-based DNA vaccine encoding T cell epitopes of Esat- 6 and FL against Mycobacterium tuberculosis infection. PLoS One 2013, 8(4):e61135.

20. Zaharoff DA, Rogers CJ, Hance KW, Schlom J, Greiner JW: Chitosan solution enhances both humoral and cell-mediated immune responses to subcutaneous vaccination. Vaccine 2007, 25(11):2085-2094.

21. Akhlaghi SP, Saremi S, Ostad SN, Dinarvand R, Atyabi F: Discriminated effects of thiolated chitosan-coated pMMA paclitaxel-loaded nanoparticles on different normal and cancer cell lines. Nanomedicine 2010, 6(5):689-697.

22. Atyabi F, Talaie F, Dinarvand R: Thiolated chitosan nanoparticles as an oral delivery system for Amikacin: in vitro and ex vivo evaluations. J Nanosci Nanotechnol 2009, 9(8):4593-4603.

23. Yousefpour P, Atyabi F, Vasheghani-Farahani E, Movahedi AA, Dinarvand R: Targeted delivery of doxorubicin-utilizing chitosan nanoparticles surface-functionalized with anti-Her2 trastuzumab. Int I Nanomed 2011, 6:1977-1990.

24. Tahamtan A, Tabarraei A, Moradi A, Dinarvand M, Kelishadi M, Ghaemi A, Atyabi F: Chitosan nanoparticles as a potential nonviral gene delivery for HPV-16 E7 into mammalian cells. Artif Cells Nanomed Biotechnol 2014 doi:10.3109/21691401.2014.893522.

25. Bivas-Benita M, Laloup M, Versteyhe S, Dewit J, De Braekeleer J, Jongert E, Borchard G: Generation of toxoplasma gondii GRA1 protein and DNA vaccine loaded chitosan particles: preparation, characterization, and preliminary in vivo studies. Int J Pharm 2003, 266(1-2):17-27.

26. Li GP, Liu ZG, Liao B, Zhong NS: Induction of Th1-type immune response by chitosan nanoparticles containing plasmid DNA encoding house dust mite allergen Der $\mathrm{p} 2$ for oral vaccination in mice. Cell Mol Immunol 2009, 6(1):45-50.

27. Khatri K, Goyal AK, Gupta PN, Mishra N, Vyas SP: Plasmid DNA loaded chitosan nanoparticles for nasal mucosal immunization against hepatitis $B$. Int J Pharm 2008, 354(1-2):235-241. 
28. Jiang L, Qian F, He X, Wang F, Ren D, He Y, Li K, Sun S, Yin C: Novel chitosan derivative nanoparticles enhance the immunogenicity of a DNA vaccine encoding hepatitis B virus core antigen in mice. J Gene Med 2007, 9(4):253-264

29. Zhao K, Shi X, Zhao Y, Wei H, Sun Q, Huang T, Zhang X, Wang Y: Preparation and immunological effectiveness of a swine influenza DNA vaccine encapsulated in chitosan nanoparticles. Vaccine 2011, 29(47):8549-8556.

30. Zhou X, Zhang X, Yu X, Zha X, Fu Q, Liu B, Wang X, Chen Y, Chen Y, Shan Y, Jin Y, Wu Y, Liu J, Kong W, Shen J: The effect of conjugation to gold nanoparticles on the ability of low molecular weight chitosan to transfer DNA vaccine. Biomaterials 2008, 29(1):111-117.

31. Klencke B, Matijevic M, Urban RG, Lathey JL, Hedley ML, Berry M, Thatcher J, Weinberg V, Wilson J, Darragh T, Jay N, Da Costa M, Palefsky JM: Encapsulated plasmid DNA treatment for human papillomavirus 16-associated anal dysplasia: a Phase I study of ZYC101. Clin Cancer Res 2002, 8(5):1028-1037.

doi:10.1186/s12929-014-0069-z

Cite this article as: Tahamtan et al:: Antitumor effect of therapeutic HPV

DNA vaccines with chitosan-based nanodelivery systems. Journal of Biomedical

Science 2014 21:69.

\section{Submit your next manuscript to BioMed Central and take full advantage of:}

- Convenient online submission

- Thorough peer review

- No space constraints or color figure charges

- Immediate publication on acceptance

- Inclusion in PubMed, CAS, Scopus and Google Scholar

- Research which is freely available for redistribution 